\title{
Compressed Sensing Based Channel Estimation for OFDM Transmission under 3GPP Channels
}

\author{
Han Wang, Wencai Du and Yong Bai \\ College of Information Science \& Technology, Hainan University, 58 Renmin Ave., \\ Haikou, Hainan 570228, China. \\ hanwang1214@gmail.com,wencai@hainu.edu.cn and bai@hainu.edu.cn
}

\begin{abstract}
A large number of pilots are utilized to acquire channel information in traditional channel estimation for Orthogonal Frequency Division Multiplexing (OFDM) system, which leads to lower spectrum efficiency. For exploiting the sparse channel characteristics of 3GPP multipath channels, we employ the Compressed Sensing (CS) approach for channel estimation. Two CS-based recovery algorithms, Orthogonal matching pursuit (OMP) algorithm and Compressive sampling matching pursuit $(\mathrm{CoSaMP})$ algorithm, are considered in this paper. The Bit error rate (BER) and Mean squared error (MSE) performance using traditional least square( $L S)$, and two CS-based algorithms are given. Simulation results demonstrate that the CoSaMP algorithm achieves best performance with fewer pilots among three algorithms under 3GPP channels.
\end{abstract}

Keywords: Compressed Sensing, Channel Estimation, OFDM, 3GPP Channel, Cosamp

\section{Introduction}

In wireless communications, multipath radio channels often leads to frequencyselective fading and serious Inter-Symbol Interference (ISI) for high speed data transmissions. The Orthogonal frequency division multiplexing (OFDM) techniques has been widely employed to combat multipath interference at high data rate. Channel estimation is essentially a process of recover channel impulse response, and it is an important part of OFDM transmission system. Rich multipath channel is usually assumed, and long training sequences/large number of pilots are required in the traditional channel estimation methods like least squares (LS) and minimum mean squared error (MMSE) [1], which reduce the frequency spectrum utilization. In reality, the wireless multipath channel exhibit sparse structure [2], such as 3GPP channels. Hence, exploiting the sparse characteristics for channel estimation can improve the spectrum utilization, and compressed sensing provides such an approach.

Compressed sensing (CS) theory was proposed by E.J.Candes, J.Romberg, T.Tao and L.Donoho [3, 4] in 2006. They have proved that if signal in an orthogonal space can be sparse represented, the signal sampling can use lower sampling frequency. With some recovery algorithm, the signal can be restored with high probability, which contrasts with the Nyquist sampling theorem. The appearance of the theory has received widespread attention in sparse signal processing [5]. In [6], the authors analyzed current application of compressed sensing to sparse channel estimation, and proposed the channel modeling method and how to insert pilot sub-carrier. The research of CS-based channel estimation include Ultra-wideband (UWB), OFDM, and Multi-input multi-output (MIMO) systems [7-9]. In [10], Matching pursuit (MP) algorithm for CS signal recovery is proposed. In [11], OMP algorithm for CS signal recovery is proposed. It improves the estimation 
accuracy, but increases the computational complexity. In [12], performance of OMP and CoSaMP (compressive sampling matching pursuit) based channel estimation in amplify and forward two way relay network (AF-TWRN) scenario is compared. CoSaMP algorithm performs iterative signal recovery form incomplete and inaccurate measurements [13]. The conclusion is that CoSaMP-based channel estimation performs similar to OMP-based channel estimation in AF-TWRN scenario.

In this paper, we investigate the CS-based channel estimation for OFDM transmission under 3GPP channels. The LS, OMP and CoSaMP algorithms are considered as the recovery algorithms for channel estimation. The BER and MSE performance of the three algorithms with different number of pilots are compared in two 3GPP channels, Pedestrian A channel and Vehicular A channel. The analysis shows that CoSaMP algorithm obtains better performance than OMP and LS algorithms in two 3GPP channel models with fewer pilots.

The rest of this paper is organized as follows. In Section 2, we introduce the CS-based OFDM transmission system model. Section 3 introduces the CS-based channel estimation theory, and describes the OMP and CoSaMP recovery algorithms. Simulation results are given and analyzed in Section 4. Finally, Section 5 concludes our work.

\section{System Model}

The CS-based block diagram of OFDM transmission system is shown in Figure 1. The input data is first modulated and converted into parallel. In this paper, we consider selecting number of $P$ sub-carriers in $N$ sub-carriers OFDM system as pilots, and the pilots are inserted into data sub-carriers at regular intervals. In order to avoid the intersymbol interference (ISI) due to multipath delay spread, a cyclic prefix (CP) of length equal or greater than the maximum expected time delay of the channel is inserted in each OFDM symbol prior to transmission. By N-point Inverse fast fourier transform (IFFT) and $\mathrm{CP}$ insertion, the symbols are transmitted. At the receiver side, the $\mathrm{CP}$ is removed, and the received data are passed to fast fourier transform (FFT) block. Then, the received symbols are extracted to channel estimator which estimates the channel frequency response and delay spread parameters.

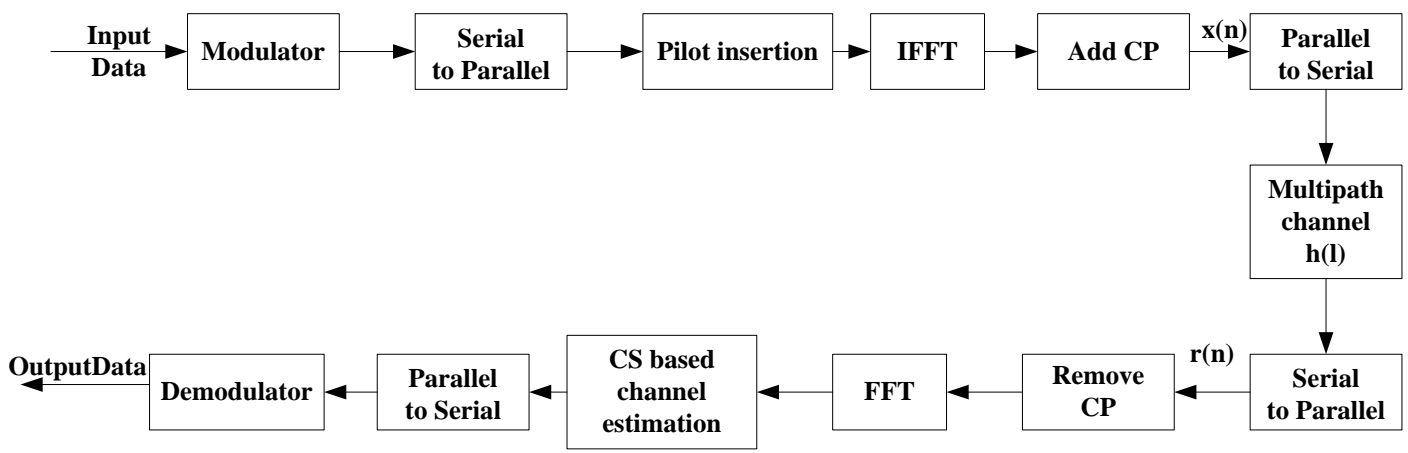

Figure 1. CS-Based OFDM Transmission Model

At the transmitter, the time-domain samples of transmission signal can be written as $x(n),(0 \leq n \leq N-1), N$ is the total number of sub-carriers. Assuming that the transmission system has good synchronization performance, the received signal, denoted by $r(n)$, is the convolution of the transmitted signal and the channel response plus noise. It is given by

$$
r(n)=\sum_{l}^{L-1} x(n-l) h(l)+z(n) 0 \leq n \leq N-1
$$


where $L$ is the number of sample-spaced channel taps, $z(n)$ is the additive white Gaussian noise (AWGN) sample with zero mean and variance of $\sigma^{2}$, and $h(l)$ is the multipath channel impulse response in time domain. The multipath channel impulse response can be described as [14]

$$
h(t)=\sum_{l=0}^{L-1} a_{l}(t) \delta\left(\tau-\tau_{l}\right)
$$

where $\tau_{l}$ is the delay of $l t h$ path and $a_{l}(t)$ is the complex amplitude of lth path, which is assumed to be wide-sense-stationary (WSS) complex Gaussian processes and independent between different paths.

\section{Compressed Sensing based Channel Estimation}

In this section, the CS theory for channel estimation is briefly described. The spare representation of the multipath channel system is provided, then we describe two CS signal recovery algorithms, OMP and CoSaMP.

CS theory mainly includes three aspects, sparse representation of signal, the measurement matrix and recovery algorithm.

Assuming that signal

$$
h=\sum_{i=1}^{N} \alpha_{i} \psi_{i}=\psi a
$$

where $\psi_{i}(i=1,2, \ldots, N)$ are base vectors, $a$ and $h$ are $N \times 1$ matrix, $\Psi$ is an $N \times N$ matrix. If the number of non-zero elements $K$ in signal $h$ far less than $N$, the signal can be thought as $K$-spares signal.

CS theory states that a $K$-spares signal $h$ can be stably recovered from linear measurement

$$
y=\theta h+z
$$

where $\theta$ is a matrix with $M$ rows and $N$ columns, $M \square N$, and $z$ is noise. The premise is that the measurement matrix $\theta$ satisfies the Restricted Isometry Property (RIP), for all $K$-spares $h$,

$$
1-\delta_{K} \leq \frac{\|\phi \mathrm{h}\|_{2}^{2}}{\|\mathrm{~h}\|_{2}^{2}} \leq 1+\delta_{K}
$$

where $0<\delta_{K}<1$ is the RIP parameter.

The received signal in (1) in Section 2 can be written in matrix form as

$$
R=X H+Z
$$

where $R=[r(0), r(1), \ldots, r(N-1)]^{T}, X=\operatorname{diag}(x(0), x(1), \ldots, x(N-1)), H=F_{N L} h$ is the multipath channel frequency response sampling values, $F_{N L}$ is part of FFT matrix with it elements at row $N$ and column $L . z$ is a $N \times N$ matrix with zero mean and variance of $\sigma^{2}$.

Here, we set $P$ as the number of pilots, $\varphi=\left(\mathrm{e}_{\mathrm{s}_{1}}, \mathrm{e}_{\mathrm{s}_{2}}, \ldots, \mathrm{e}_{\mathrm{s}_{P}}\right)$ is a $P \times N$ pilot selection matrix, which is used to select the pilots position from total $N$ sub-carriers. $\mathrm{s}_{i}(i=1,2, \ldots, P)$ is the position of the $i$ pilot. Converting $\varphi$ to (6) as

$$
R_{P}=X_{F} F_{p} h+z_{P}
$$


where $R_{P}=\varphi R$ is received pilot signal, $z_{P}=\varphi z$, they are both $P$ column vector. $X_{P}=\varphi X \varphi^{T}$ is a diagonal matrix, the diagonal elements are pilot values, $F_{P}=\varphi F_{N L}$.

Assuming that $T=X_{P} F_{P}$, converting (7) to

$$
R_{P}=T h+z_{P}
$$

where $h$ is multipath channel impulse response, $R_{P}$ and $T$ can be obtained in the transmission process, then the CS recovery algorithm can be used to recover $h$.

The key of recovery algorithm is how to accurately recover original high dimensional data from low dimensional data which are from compressed sensing. Many recovery algorithms like basis pursuit (BP), orthogonal matching pursuit (OMP) and compressive sampling matching pursuit (CoSaMP) have been studied for recovery of the vector $\theta$. In this paper, we utilize OMP and CoSaMP algorithms as the recovery algorithms to estimate channels. The OMP algorithm and CoSaMP algorithm are shown as follows.

\section{OMP Algorithm}

Input: residual $\boldsymbol{r}$, linear measurement $\boldsymbol{y}$, measurement matrix $\boldsymbol{Y}$, recovery matrix $\boldsymbol{T}$, sparse $\boldsymbol{K}$

Output: channel estimator $\boldsymbol{h}_{\boldsymbol{e}}$ Initialize: $\mathbf{T}=\phi, \mathbf{Y}=\phi, \mathbf{r}=\mathbf{y}$, iterations $\boldsymbol{i}=1: 1: \boldsymbol{K}$

The absolute value of inner product of $\boldsymbol{Y}^{\boldsymbol{T}}$ and $\boldsymbol{r}: \boldsymbol{U}=\operatorname{sum}\left(\operatorname{abs}\left(\boldsymbol{Y}^{\boldsymbol{T}} * \boldsymbol{r}\right)\right)$, where $*$ is inner product operation;

Record the position of $\boldsymbol{U}$ and its value: $[\boldsymbol{V}, \boldsymbol{P}]=\max (\boldsymbol{U})$;

Measurement matrix $\boldsymbol{Y}$ expansion: $\boldsymbol{Y}=[\boldsymbol{Y}, \boldsymbol{T}(:, \boldsymbol{P})]$;

Signal approximation with least squares: $\boldsymbol{h}_{e}=\left(\boldsymbol{Y}^{\boldsymbol{T}} * \boldsymbol{Y}\right)^{-1} * \boldsymbol{Y}^{\boldsymbol{T}} * \boldsymbol{r}$;

Update residual $: \boldsymbol{r}=\boldsymbol{y} \boldsymbol{Y} * \boldsymbol{h}_{\boldsymbol{e}}$;

Record the position of projection: $\boldsymbol{Y}_{s}(:, \boldsymbol{i})=\boldsymbol{Y}(:, \boldsymbol{P})$;

End iteration

\section{Cosamp Algorithm}

Input: residual $\boldsymbol{r}$, linear measurement $\boldsymbol{y}$, measurement matrix $\boldsymbol{Y}$, recovery matrix $\boldsymbol{T}$, sparse $\boldsymbol{K}$

Output: channel estimator $\boldsymbol{h}_{\boldsymbol{e}}$

Initialize: $\mathbf{T}=\phi, \mathbf{Y}=\phi, \mathbf{r}=\mathbf{y}$, iterations $\boldsymbol{i}=1: 1: 2 \boldsymbol{K}$

The absolute value of inner product of $\boldsymbol{Y}^{\boldsymbol{T}}$ and $\boldsymbol{r}: \boldsymbol{U}=\operatorname{sum}\left(\operatorname{abs}\left(\boldsymbol{Y}^{\boldsymbol{T}} * \boldsymbol{r}\right)\right)$, where $*$ is inner product operation;

Sort the value: $[\boldsymbol{V}, \boldsymbol{P}]=\operatorname{sort}\left(\boldsymbol{U},{ }^{\prime} \operatorname{desec}\right.$ end $\left.^{\prime}\right)$;

Record the position for current iteration: $\boldsymbol{S}_{\boldsymbol{P}}=\operatorname{union}(\boldsymbol{P}, \boldsymbol{Y})$;

Current measurement matrix: $\boldsymbol{Y}=\boldsymbol{Y}\left(: ; S_{P}\right)$;

Signal approximation with least squares: $\boldsymbol{h}_{e}=\left(\boldsymbol{Y}^{\boldsymbol{T}} * \boldsymbol{Y}\right)^{-1} * \boldsymbol{Y}^{\boldsymbol{T}} * \boldsymbol{r}$;

Update residual : $\boldsymbol{r}=\boldsymbol{y} \boldsymbol{Y} * \boldsymbol{h}_{\boldsymbol{e}}$;

Record the position of projection: $\boldsymbol{Y}_{s}(:, \boldsymbol{i})=\boldsymbol{Y}(:, \boldsymbol{P})$; 
End iteration

\section{Simulation Results and Analysis}

In the simulated transmission system, the total sub-carriers is $N=256$, including pilots sub-carriers. The length of CP is 20. QPSK modulation scheme is used. Monte carlo simulations are performed over 100 realizations of random channel vectors. We investigate the performance of CS-based OFDM transmission under 3GPP Pedestrian A channel and Vehicular A channel. The two channel models are show in Table 1 and Table 2 , respectively.

Table 1. Channel Delay Profiles of 3GPP Pedestrian A Channel

\begin{tabular}{|l|l|l|}
\hline Tap & Relative Delay(ns) & $\begin{array}{l}\text { Average relative } \\
\text { power(dB) }\end{array}$ \\
\hline 1 & 0 & 0 \\
\hline 2 & 110 & -9.7 \\
\hline 3 & 190 & -19.2 \\
\hline 4 & 410 & -22.8 \\
\hline
\end{tabular}

Table 2. Channel Delay Profiles of 3gpp Vehicular A Channel

\begin{tabular}{|l|l|l|}
\hline Tap & Relative Delay(ns) & $\begin{array}{l}\text { Average relative } \\
\text { power(dB) }\end{array}$ \\
\hline 1 & 0 & 0 \\
\hline 2 & 310 & -1.0 \\
\hline 3 & 710 & -9.0 \\
\hline 4 & 1090 & -10.0 \\
\hline 5 & 1730 & -15.0 \\
\hline 6 & 2510 & -20.0 \\
\hline
\end{tabular}

Figure 2 and Figure 3 show the variation of BER and MSE (Mean Squared Error) with different estimation algorithms (LS, OMP, CoSaMP) for different pilots in Pedestrian A channel, respectively. The sparse value $K=4$. In the simulation, LS and OMP uses 32 pilots, CoSaMP uses 16 pilots. It is seen that OMP outperforms LS with the same number of pilots, and CoSaMP can obtain the best performance in the three algorithms with fewer pilots. 


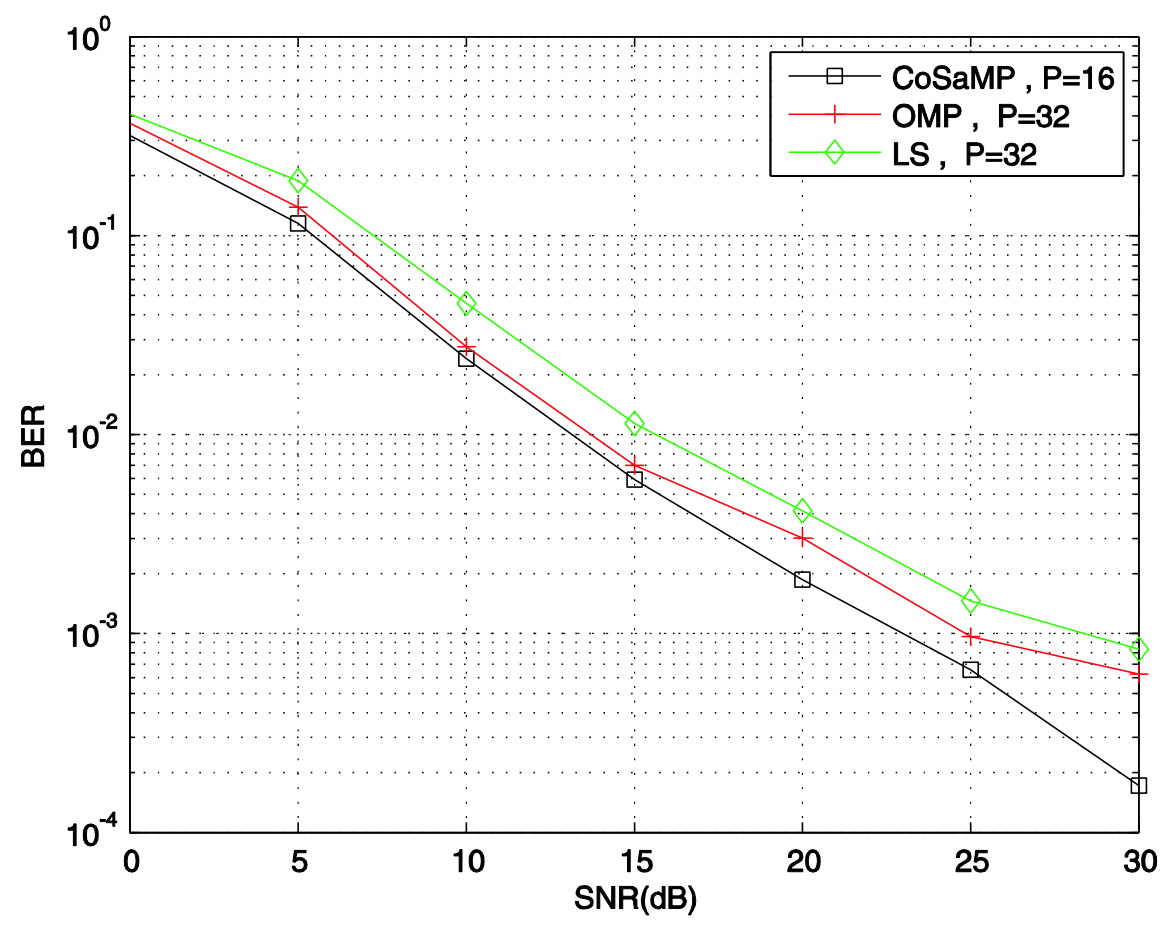

Figure 2. BER Performance in Pedestrian A Channel

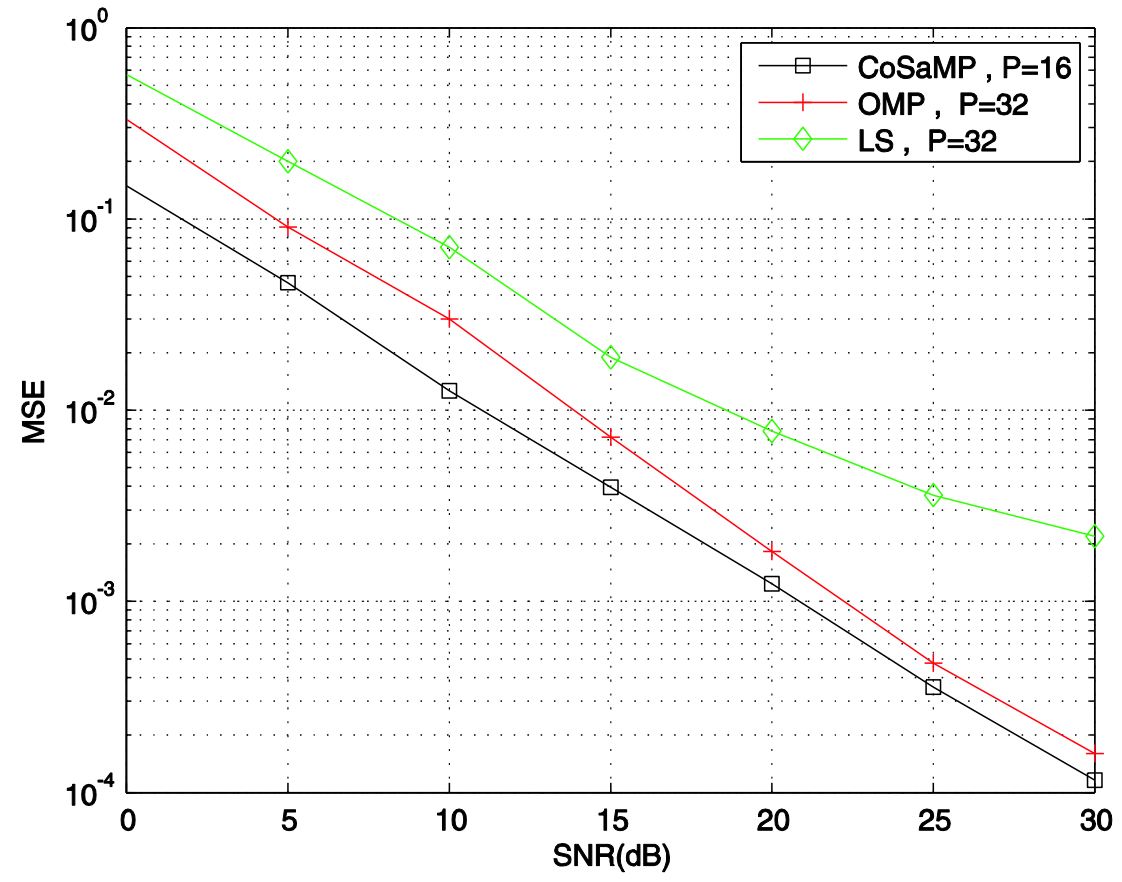

Figure 3. MSE Performance in Pedestrian A Channel

In Figure 2, when the SNR is above $15 \mathrm{~dB}$, BER performance of CoSaMP becomes significantly better than OMP. For example, with the same $B E R=10^{-2}$, CoSaMP can gain 
about $0.5 \mathrm{~dB}$ than OMP. With $B E R=10^{-3}, \mathrm{CoSaMP}$ can gain about $2 \mathrm{~dB}$ than OMP. In Figure 3, MSE values of CoSaMP are less than OMP about $3 \mathrm{~dB}$.

Figure 4 and Figure 5 depict the variation of BER and MSE with different estimation algorithms (LS, OMP, CoSaMP) for different pilots in Vehicular A channel, respectively. The sparse value $K=6$. The trends of BER and MSE curves are the same as that in Figure 2 and Figure 3. It can be observed that CoSaMP achieves best BER and MSE performance in Vehicular A channel.

Comparing Figure 2 and Figure 4, the superiority of CoSaMP compared with OMP is more obvious. For example, with the same $B E R=10^{-2}$, CoSaMP gets about $2.5 \mathrm{~dB}$ gain than OMP, while $B E R=10^{-3}$, CoSaMP gain about $7 \mathrm{~dB}$ than OMP. Moreover, the MSE values of CoSaMP are less than that of OMP about $5 \mathrm{~dB}$. Hence, CoSaMP can obtain more gain in Vehicular A channel.

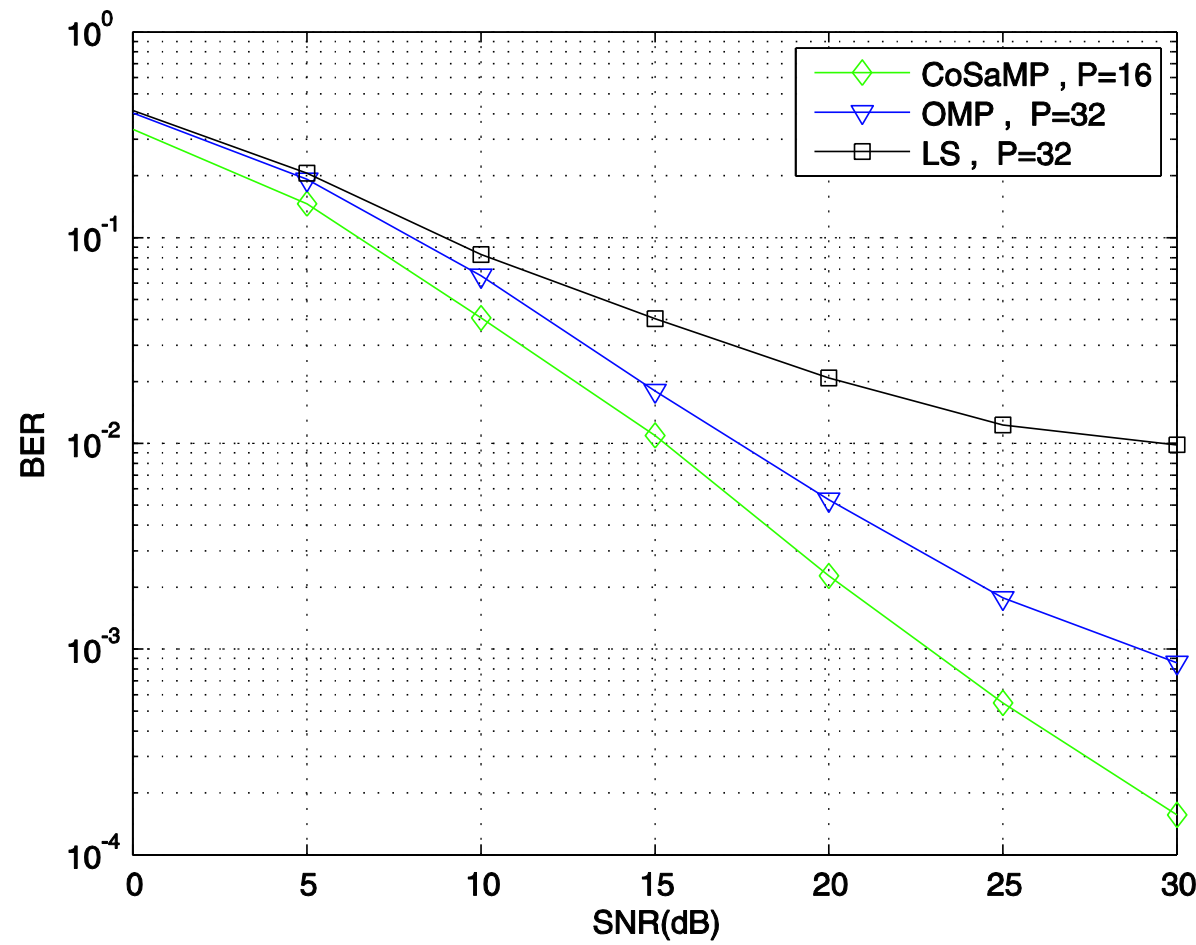

Figure 5. MSE Performance in Vehicular A Channel

From the simulation results, it is seen that the BER of CoSaMP decreases from $\mathrm{SNR}=15$ to $30 \mathrm{~dB}$ and the MSE performance is also decreased with the number of multipath increases, but it achieves the better performance than other two algorithms.

\section{Conclusion}

In this paper, we investigated the compressed sensing (CS)-based channel estimation for OFDM transmission in 3GPP channel scenarios, 3GPP Pedestrian A channel and Vehicular A channel. In OFDM system, we insert pilot sub-carrier at regular intervals, and cyclic prefix (CP) length is fixed. The BER and MSE performance are compared using three different recovery algorithms, LS, OMP and CoSaMP. The CoSaMP algorithm can achieves better performance with fewer pilots than OMP algorithm. Based on the simulation results, it is concluded that CoSaMP algorithm outperforms OMP algorithm for channel estimation of OFDM transmission system under Pedestrian A 
channel estimation and Vehicular A channel. Specifically, the improvement of CoSaMP algorithm is more significant in Vehicular A channel than in Pedestrian A channel.

\section{Acknowledgements}

This paper was supported by the National Natural Science Foundation of China (Grant No. 61062006 and Grant No. 61261024 and No.61162010) and the Special Social Service Project Fund of Hainan University, China (Grant No.HDSF201301).

\section{References}

[1] L. Kewen and X. Ke, "Research of MMSE and LS channel estimation in OFDM systems", The $2^{\text {nd }}$ international conference on information science and engineering (ICISE2010), (2010), pp. 2308-2311.

[2] J. L. Pardes, G. R. Arce and Z. M. Wang, "Ultra-wideband compressed sensing: channel estimation", IEEE Journal of Selected Topics in Signal Processing, vol. 1, no. 3, (2007), pp. 383-395.

[3] E. Candes and E, Romberg J, "Quantitative robust uncertainty principles and optimally sparse decompositions", Foundations of Computational Mathematics, vol. 6, no. 2, (2006), pp. 227-254.

[4] E. Candes, J. Romberg and T. Tao, "Robust uncertainty principles: exact signal recovery from highly incomplete frequency information", IEEE Transaction on Information Theory, vol. 52, no. 4, (2006), pp. 489-509.

[5] Z. Domoosh, "On the recovery of sequences of sparse signals-The Weighted-CS", IEEE International Conference on Acoustics, Speech and Signal Processing (ICASSP2013), (2013), pp. 196-202.

[6] C. R. Berger, Z. Wang and J. Huang, "Application of compressive sensing to sparse channel estimation", IEEE Communications Magazine, vol. 48, no. 11, (2010), pp. 164-174.

[7] E. Lagunas, "Sparse channel estimation based on compressed sensing for ultra wideband systems", IEEE International Conference on Ultra-Wideband (ICUWB2011), (2011), pp. 365-369.

[8] J. Meng, W. Yin and Z. Han, "Compressive sensing based high-resolution channel estimation for OFDM system”, IEEE Journal of Selected Topics in Singal Processing, vol. 6, no.1, (2012), pp. 15-25.

[9] Z. B. Xie, "A sparsity enhanced channel estimation algorithm based on compressed sensing in MIMOOFDM systems", Journal of Electronics and Information Technology, vol. 35, no. 3, (2013), pp. 665-670.

[10] S. F. Cotter and B. D. Rao, "Sparse channel estimation via matching pursuit with application to equalization", IEEE Transaction on Communications, vol. 50, no. 3, (2002), pp. 374-377.

[11] J. Tropp and A. Gilbert, "Signal recovery from random measurements via orthogonal matching pursuit", IEEE Transactions on Information Theory, vol. 53, no. 12, (2007), pp. 4655-4666.

[12] Y. Gaur and V. K. Chakka, "Performance comparison of OMP and CoSaMP based channel estimation in AF-TWRN scenario", 2012 Third International Conference on Computer and Communication Technology(ICCCT2012), (2012), pp. 186-190.

[13] J. A. Troup and D. Needell, "CoSamp: iterative signal recovery form incomplete and inaccurate measurements", Applied and Computational Harmonic Analysis, vol. 26, no. 3, (2009), pp. 301-321.

[14] W. Liu, X. Liao and Y. Bai, "SC-FDE Transmission with Length-Adaptive Cyclic Prefix Aided by Delay Spread Estimation”, Wireless Personal Communications, vol. 79, no. 3, (2014), pp. 2059-2067.

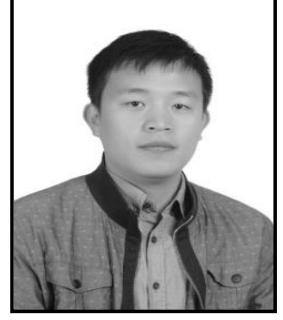

Han Wang received his B.S. degree in electrical engineering from Hubei University of Nationalities, China, in 2009 and the M.S. degree in information and communication system from Hainan University, Haikou, China, in 2013. He has worked in China Mobile Jiangxi branch as a network engineer for one year. Now, he is pursuing the Ph.D. degree with the Department of College of Information Science \& Technology in Hainan University. His research interests include maritime communications and information theory. 


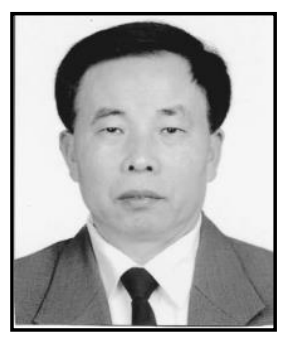

Wencai Du received the B.S. degree from Peking University, China, two M.S. Degrees from ITC, The Netherlands, and Hohai University, China, respectively, and Ph.D. degree from South Australia University, Australia. He was a Post-doctor Fellow in Israel Institute of Technology (IIT), Haifa, Israel. He is Dean of College of Information Science \& Technology at Hainan University and Director of Maritime Communication and Engineering of Hainan province. He has authored or coauthored 18 books and more than 80 scientific publications. He is currently members of the Editorial Board of Inverts Journal of Science and Technology, India. He has taken services on many professional conferences, including Conference Chair of IEEE/ACIS ICIS 2011, Conference Co-Chair of IEEE/ACIS SNPD 2010, London, Conference Chair of IEEE/ACIS SERA 2009, and Program Chair of IEEE/ACIS SNPD 2009, Daegu, Korea. His research interests include several aspects of Information Technology and Communication (ITC), including computer network and maritime communications.

Yong Bai received his B.S. degree from Xidian University, China, in 1992, and M.S. degree from Beijing University of Posts and Telecommunications (BUPT), China, in 1995, and Ph.D. degree from Rutgers-The State University of New Jersey in 2001. He was with Packet Video Corporation from 2000 to 2002. He was with Motorola from 2002 to 2004. He was with CEC Wireless from 2004 to 2005. $\mathrm{He}$ was a senior researcher at DOCOMO Beijing Communication Labs from 2005 to 2009 . He is a professor at College of Information Science \& Technology, Hainan University since 2010. He acted as the Lead Guest Editor for EURASIP Journal on Wireless Communications and Networking, Special Issue on Topology Control in Wireless Ad Hoc and Sensor Networks. His current research interests include mobile communications, and maritime communications. He is a member of the IEEE. 
International Journal of Future Generation Communication and Networking Online Vol. 9, No. 4, (2016) 